\title{
P-glycoprotein Inhibition Increases the Brain Distribution and Antidepressant-Like Activity of Escitalopram in Rodents
}

\author{
Fionn E O'Brien 1,2,3, Richard M O'Connor ${ }^{3}$, Gerard Clarke ${ }^{1,4}$, Timothy G Dinan ${ }^{1,4}$, Brendan T Griffin ${ }^{2}$ and \\ John F Cryan*, I,3 \\ 'Alimentary Pharmabiotic Center, University College Cork, Cork, Ireland; ${ }^{2}$ Pharmacodelivery Group, School of Pharmacy, University College Cork, \\ Cork, Ireland; ${ }^{3}$ Department of Anatomy and Neuroscience, University College Cork, Cork, Ireland; ${ }^{4}$ Department of Psychiatry, University College \\ Cork, Cork, Ireland
}

\begin{abstract}
Despite the clinical prevalence of the antidepressant escitalopram, over $30 \%$ of escitalopram-treated patients fail to respond to treatment. Recent gene association studies have highlighted a potential link between the drug efflux transporter P-glycoprotein (P-gP) and response to escitalopram. The present studies investigated pharmacokinetic and pharmacodynamic interactions between P-gP and escitalopram. In vitro bidirectional transport studies revealed that escitalopram is a transported substrate of human P-gp. Microdialysisbased pharmacokinetic studies demonstrated that administration of the P-gP inhibitor cyclosporin A resulted in increased brain levels of escitalopram without altering plasma escitalopram levels in the rat, thereby showing that P-gp restricts escitalopram transport across the blood-brain barrier (BBB) in vivo. The tail suspension test (TST) was carried out to elucidate the pharmacodynamic impact of P-gP inhibition on escitalopram effect in a mouse model of antidepressant activity. Pre-treatment with the P-gp inhibitor verapamil enhanced the response to escitalopram in the TST. Taken together, these data indicate that P-gP may restrict the BBB transport of escitalopram in humans, potentially resulting in subtherapeutic brain concentrations in certain patients. Moreover, by verifying that increasing escitalopram delivery to the brain by P-gp inhibition results in enhanced antidepressant-like activity, we suggest that adjunctive treatment with a P-gp inhibitor may represent a beneficial approach to augment escitalopram therapy in depression.

Neuropsychopharmacology (2013) 38, 2209-2219; doi:I0.1038/npp.2013.120; published online 5 June 2013
\end{abstract}

Keywords: P-glycoprotein; escitalopram; antidepressant; blood-brain barrier; microdialysis; treatment-resistant depression

\section{INTRODUCTION}

Escitalopram, a selective serotonin reuptake inhibitor (SSRI), is among the most commonly prescribed and clinically important antidepressants. However, over $30 \%$ of escitalopram-treated depressed patients fail to respond to treatment, with a full remission achieved in only $50 \%$ of cases (Kennedy et al, 2006). Recent clinical studies have found associations between single-nucleotide polymorphisms (SNPs) in $A B C B 1$, the gene encoding the multidrug efflux transporter P-glycoprotein (P-gp) in humans, and escitalopram response (Lin et al, 2011; Singh et al, 2012). Given that drug efflux by P-gp at the blood-brain barrier (BBB) can prevent therapeutic concentrations of centrallyacting drugs from being achieved in the brain (Loscher and Potschka, 2005), this genetic association may indicate that escitalopram efflux by P-gp at the $\mathrm{BBB}$ contributes to the high prevalence of treatment failure (O'Brien et al, 2012b).

*Correspondence: Professor JF Cryan, Department of Anatomy and Neuroscience, University College Cork, Cork, Ireland, Tel: +353 21 420 5426, Fax: +353 21420 5479, E-mail: j.cryan@ucc.ie

Received 20 March 2013; revised 27 April 2013; accepted 7 May 2013; accepted article preview online I4 May 2013
Therefore, findings from these clinical pharmacogenetic studies proffer the intriguing possibility that augmentation of failed escitalopram treatment with adjunctive P-gp inhibition therapy may represent a novel approach to overcome treatment-resistant depression in certain patients. Furthermore, in light of recent regulatory guidance, which has recommended the limitation of escitalopram doses used clinically because of the risk of QT prolongation, especially in the elderly (Medicines and Healthcare Products Regulatory Agency, 2011), it would be advantageous if it were possible to selectively enhance escitalopram delivery to the brain by P-gp inhibition. This could facilitate escitalopram dose reduction while maintaining therapeutic response, thereby helping to minimize problems associated with peripheral side effects.

However, several questions remain to be answered before this potential therapeutic strategy becomes realized. For example, it is unknown if escitalopram is a transported substrate of human P-gp or if P-gp efflux restricts the transport of escitalopram across the BBB. Moreover, it is unknown if any putative enhancement of escitalopram brain levels by P-gp inhibition would result in an augmentation of its pharmacodynamic activity. Although several studies have demonstrated that P-gp restricts the brain distribution of various antidepressants in vivo 
(Uhr et al, 2000, 2003, 2007, 2008; Uhr and Grauer, 2003; Doran et al, 2005; Ejsing and Linnet, 2005; Clarke et al, 2009; Karlsson et al, 2010; Bundgaard et al, 2012; O’Brien et al, 2012a), it has not yet been determined if increasing brain levels of any antidepressant due to P-gp knockout or P-gp inhibition results in an augmentation of pharmacodynamic effect in an appropriate animal model of antidepressant activity.

This study was designed to address these outstanding questions, using a combination of in vitro and in vivo approaches to investigate pharmacokinetic and pharmacodynamic interactions between P-gp and escitalopram. In vitro bidirectional transport studies, using cell lines that express human P-gp (Figure 1a), represent the gold standard for the identification of transported substrates of human P-gp ( O'Brien et al, 2012b). This is particularly important considering well-established species differences in P-gp specificity (Yamazaki et al, 2001; Katoh et al, 2006; Baltes et al, 2007; Syvanen et al, 2009). The integrated microdialysis approach adopted in pharmacokinetic studies facilitated the simultaneous and repeated monitoring of plasma and brain levels of escitalopram before and after administration of a P-gp inhibitor under steady-state conditions. This approach gives a unique insight into the effect of P-gp inhibition on escitalopram transport across the BBB. Pharmacodynamic studies were carried out to determine if increasing the delivery of escitalopram to the brain by inhibition of P-gp would result in enhanced antidepressant-like activity. To this end, the tail suspension test (TST), which is among the most commonly used and well-validated preclinical models for the assessment of antidepressant activity (Cryan et al, 2005), was conducted.

\section{MATERIALS AND METHODS}

\section{Drugs and Chemicals}

Acetonitrile, potassium dihydrogen phosphate and orthophosphoric acid were obtained from Fisher Scientific (Ireland). Heparin sodium solution (Wockhardt UK, UK) and cyclosporin A (CsA; Sandimmun) were purchased from Uniphar Group (Ireland). Escitalopram oxalate was purchased from Discovery Fine Chemicals (Dorset, UK). Verapamil hydrochloride and imipramine hydrochloride were obtained from Sigma-Aldrich (Ireland), as were all other chemicals, reagents, and materials, unless otherwise stated.

\section{In Vitro Bidirectional Transport Studies}

Transport experiments were conducted in MDCKII-WT and MDCKII-MDR1 cells, obtained from the Laboratory of Prof Piet Borst (Netherlands Cancer Institute, Amsterdam, a

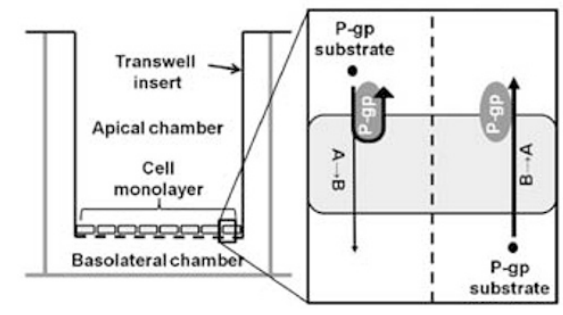

b

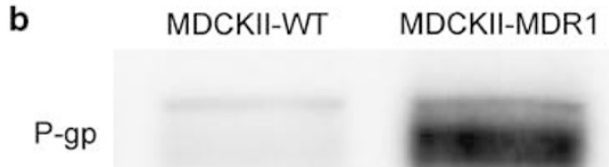

B-actin
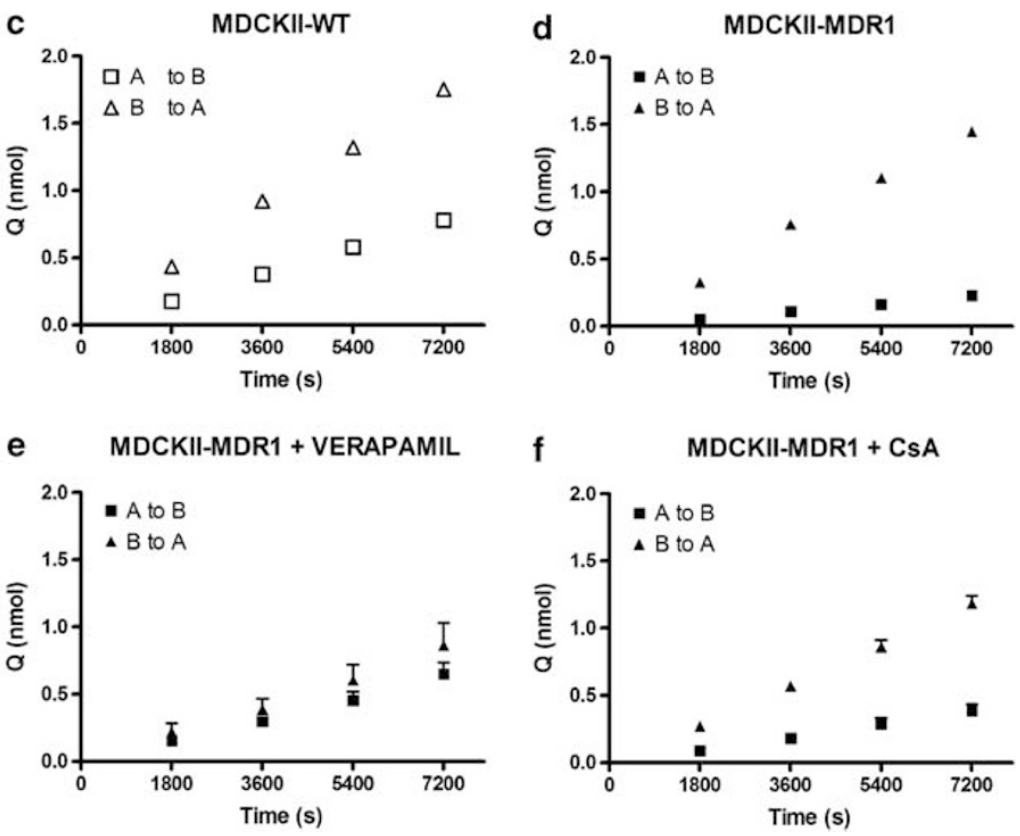

Figure I In vitro bidirectional transport studies. (a) Schematic illustrating the in vitro bidirectional transport assay. MDCKII-MDRI cells express human P-glycoprotein (P-gp) in a polarized manner at the apical membrane only, when cultured on a transwell support. When substrates of human P-gp are added to the apical chamber, their transport across the monolayer of cells is restricted by P-gP efflux, thereby reducing their apical-to-basolateral (A $\rightarrow B$ ) permeability. When substrates of human P-gp are added to the basolateral chamber, P-gp does not limit their transport across the monolayer as it is not expressed at the basolateral membrane. Therefore, human P-gp substrates will have greater permeability in the basolateral-to-apical (B $\rightarrow$ A) direction than in the $A \rightarrow B$ direction, and directional efflux is attenuated by coincubation of a P-gp inhibitor. Bidirectional transport studies are also carried out in MDCKII-WT cells, which do not express human P-gp, to determine the influence of endogenous transporters, including canine P-gp, on drug permeability. (b) Representative image of western blot demonstrating over 4.5-fold greater expression of P-gP in MDCKII-MDRI cells than MDCKII-WT cells. ( $c$ and d) Escitalopram accumulation in receiver chamber over time in MDCKII-WT and MDCKII-MDRI cells. Escitalopram crossed the monolayer to a greater extent in the $B \rightarrow A$ direction than $A \rightarrow B$ direction in both MDCKII-WT (c) and MDCKII-MDRI (d) cells, indicating net efflux in both cell lines. However, the magnitude of the efflux effect was substantially greater in MDCKII-MDRI cells, highlighting a role for human P-gp. (e and f) Escitalopram accumulation in receiver chamber over time in MDCKII-MDRI cells with coincubation of the P-gp inhibitors verapamil (e) or cyclosporin A (CsA) (f). Coincubation with either P-gp inhibitor attenuated the directional efflux of escitalopram, thereby confirming that escitalopram is a transported substrate of human P-gP (mean \pm SEM; $n=3$ in all experiments). WT, wild type. 
The Netherlands), as described in detail in Supplementary Information. The MDCKII-MDR1 cell line is transfected with $A B C B 1$ and expresses human P-gp on the apical membrane in a polarized manner when cultured on an appropriate transwell support (Pastan et al, 1988). Therefore, transported substrates of human P-gp will have lower permeability in the apical-to-basolateral $(\mathrm{A} \rightarrow \mathrm{B})$ direction than in the basolateral-to-apical direction $(\mathrm{B} \rightarrow \mathrm{A})$ due to the effect of P-gp efflux at the apical membrane, exhibiting a $(\mathrm{B} \rightarrow \mathrm{A}) /(\mathrm{A} \rightarrow \mathrm{B}$ ) transport ratio $(\mathrm{TR}) \geq 2$ (Polli et al, 2001; Zhang et al, 2006). As the MDCKII-MDR1 cell line also expresses endogenous canine kidney transporters, the TR in MDCKII-MDR1 cells can be compared with the TR in wildtype (WT) MDCKII-WT cells to yield a corrected TR (cTR), which in theory isolates the effect of human P-gp. A cTR value of $\geq 1.5$ indicates that the test compound is a transported substrate of human P-gp (Schwab et al, 2003). However, transfection with $A B C B 1$ may alter the expression of endogenous transporters (Kuteykin-Teplyakov et al, 2010). Therefore, it is important to repeat transport experiments with inhibition of P-gp to confirm that P-gp is responsible for net efflux in MDCKII-MDR1 cells. The P-gp inhibitors verapamil $(200 \mu \mathrm{M})$ and CsA $(25 \mu \mathrm{M})$ were used in this study as described previously (Tang et al, 2002; Taub et al, 2005). Expression of P-gp was confirmed by western blot as described in Supplementary Information.

\section{In Vivo Pharmacokinetic Studies}

Animals. Male Sprague-Dawley rats (Harlan Laboratories, UK), weighing $250-320 \mathrm{~g}$, were used (total $n=10$ ). Animals were group-housed 4-6 animals per cage and maintained on a $12 \mathrm{~h}$ light/dark cycle (lights on at 0800 hours) with food and water ad libitum. Room temperature was controlled at $22 \pm 1{ }^{\circ} \mathrm{C}$. All procedures were carried out in accordance with EU directive 89/609/EEC and approved by the Animal Experimentation and Ethics Committee of University College Cork.

Microdialysis probe construction and calibration. Microdialysis probes were constructed as described previously (O'Brien et al, 2012a). In vitro probe recovery values ranged from 5.64 to $8.28 \%$ for escitalopram. Dialysate values were not corrected to account for in vitro recovery, as the diffusion properties of compounds in brain tissue are likely different from in vitro conditions. Rather, dialysate values are expressed as a percentage of steady-state concentration, calculated independently for each individual animal.

Surgical procedures. To facilitate intravenous and intraarterial drug administration, as well as the collection of serial blood samples, the jugular vein and carotid artery of each rat were catheterized using standard surgical techniques, and microdialysis probes were inserted into the prefrontal cortex (PFC: $2.7 \mathrm{~mm}$ anterior and $0.7 \mathrm{~mm}$ lateral to bregma, lowered $5 \mathrm{~mm}$ from dura; Paxinos and Watson, 1998), as described previously (O'Brien et al, 2012a). After surgery, rats were single-housed in cylindrical plexiglass containers with the probe inlet connected to a fluid swivel (Instech Laboratories, Plymouth Meeting, PA, USA). Artificial cerebrospinal fluid (147 mM NaCl, $1.7 \mathrm{mM} \mathrm{CaCl}_{2}$,
$0.9 \mathrm{mM} \mathrm{MgCl}_{2}$, and $4 \mathrm{mM} \mathrm{KCl}$ ) was perfused through each microdialysis probe at a rate of $1.5 \mu \mathrm{l} / \mathrm{min}$. Rats were allowed to recover overnight before sampling on the following day, during the optimal postsurgical period (de Lange et al, 2000).

Experimental design. Rats were separated into two groups: escitalopram plus vehicle (Escit + VEH) and escitalopram plus CsA (Escit + CsA; $n=5$ per group). To achieve and maintain steady-state levels of escitalopram (Bundgaard et al, 2007), a bolus dose of escitalopram $(6 \mathrm{mg} / \mathrm{kg}$ intravenously) was administered to all rats at time $(t)=0 \mathrm{~min}$, followed by a continuous escitalopram infusion $(4 \mathrm{mg} / \mathrm{kg}$ per $\mathrm{h}$ intravenously). Rats in the Escit $+\mathrm{CsA}$ group were treated with the P-gp inhibitor CsA ( $25 \mathrm{mg} / \mathrm{kg}$ intra-arterially) (O'Brien et al, 2012a) after steady-state escitalopram levels had been achieved at $t=120 \mathrm{~min}$. Rats in the Escit $+\mathrm{VEH}$ group received the same volume $(2 \mathrm{ml} / \mathrm{kg})$ of vehicle $(1 / 12$ ethanol:2/12 Cremophor EL:9/12 saline) at $t=120 \mathrm{~min}$. Microdialysis samples were collected at 20 min intervals, and stored at $-80^{\circ} \mathrm{C}$ until analyzed by HPLC. Plasma samples $(\sim 250 \mu \mathrm{l})$ were collected at seven time points: one before escitalopram administration (blank) and one at 10,40,80, 120,160 , and $220 \mathrm{~min}$ after escitalopram bolus administration, and stored at $-80^{\circ} \mathrm{C}$ until extraction for HPLC analysis. At the conclusion of the experiment $(t=220 \mathrm{~min})$, rats were euthanized by intra-arterial administration of sodium pentobarbital. Brains were subsequently removed and dissected. The right hemisphere was taken for histological verification of probe placement. The PFC and hippocampus were taken from the contralateral hemisphere to determine escitalopram concentration in brain tissue at the termination of the experiment. Escitalopram was extracted from plasma and brain tissue using a liquidliquid extraction technique described in Supplementary Information.

\section{In Vivo Pharmacodynamic Studies}

Tail suspension test. The TST, one of the most widely used assays for assessing antidepressant activity in rodents (Cryan et al, 2005), was carried out using male C57BL/ 6JOlaHsd mice (Harlan Laboratories; 6-8 weeks old; total $n=67$ ), as described previously (O'Connor and Cryan, 2013). This allowed an assessment of the pharmacodynamic impact of pre-treatment with the P-gp inhibitor verapamil on escitalopram activity. Briefly, after a 5- to 7-day acclimation period, mice were pre-treated with either the P-gp inhibitor verapamil $(20 \mathrm{mg} / \mathrm{kg}$ intraperitoneally) or saline $1 \mathrm{~h}$ before administration of either escitalopram (0.1 or $1 \mathrm{mg} / \mathrm{kg}$ intraperitoneally) or saline. At $30 \mathrm{~min}$ after the second injection, mice were individually suspended by the tail from a horizontal bar using adhesive tape. Six-minute test sessions were recorded by a video camera and subsequently scored by a trained observer blind to the treatment groups. The amount of time spent immobile by the animal was recorded. Brain tissue (PFC and hippocampus) was harvested from each animal $10 \mathrm{~min}$ after commencement of the TST and brain escitalopram and verapamil levels were determined using the extraction procedure described in the Supplementary Information. 
Locomotor activity. To investigate potential locomotor effects, which would confound analysis of behavioral data from the TST, the impact of drug treatment on locomotor activity was assessed as described previously (O'Connor and Cryan, 2013). A separate cohort of mice were pre-treated with either the P-gp inhibitor verapamil $(20 \mathrm{mg} / \mathrm{kg}$ intraperitoneally) or saline $1 \mathrm{~h}$ before administration of either escitalopram $(0.1 \mathrm{mg} / \mathrm{kg}$ intraperitoneally) or saline, thereby resulting in four groups. At $30 \mathrm{~min}$ after the second injection, mice were individually placed in activity monitoring units $\left(27 \times 21 \mathrm{~cm}^{2}\right)$. The activity was then monitored via beam breaks for $60 \mathrm{~min}$.

\section{HPLC Analysis}

Samples were analyzed by HPLC using a reversed-phase Luna $3 \mu \mathrm{m} \mathrm{C18}(2) 150 \times 2 \mathrm{~mm}$ column (Phenomenex). The HPLC method was adapted from previously described methods (Frahnert et al, 2003; Unceta et al, 2011). Briefly, the mobile phase consisted of a mixture of $25 \mathrm{mM}$ potassium dihydrogen phosphate $(25 \mathrm{mM}, \mathrm{pH} 7$, with $4 \mathrm{~N}$ $\mathrm{NaOH})$ and HPLC grade acetonitrile $(56: 44)$. Compounds were eluted isocratically over a 15 min runtime at a flow rate of $0.4 \mathrm{ml} / \mathrm{min}$ after a $20 \mu \mathrm{l}$ injection. The UV detector was set to $230 \mathrm{~nm}$ and the fluorescent detector was set to $249 \mathrm{~nm}$ excitation and $302 \mathrm{~nm}$ emission. Escitalopram, imipramine (internal standard), and verapamil were identified and quantified based on their characteristic retention times and peak heights as determined by standard injections, which were run at regular intervals during sample analysis. The limit of quantification (LOQ) for escitalopram was $6.25 \mathrm{ng} / \mathrm{ml}$. The coefficients of variation (\% CV) for the HPLC method were $9.02,2.04$ and $0.34 \%$ at $6.25 \mathrm{ng} / \mathrm{ml}$ (LOQ), $20 \mathrm{ng} / \mathrm{ml}$ (typical of microdialysis samples) and $1000 \mathrm{ng} / \mathrm{ml}$ (typical of extracted plasma or brain samples), respectively.

\section{Data Analysis and Statistical Procedures}

All statistical analyses were carried out using standard commercial software (SPSS Statistics, version 20.0.0; SPSS, Chicago, IL). Plasma and dialysate concentration $v s$ time profiles were analyzed using one-way repeated-measures ANOVA. Where significant overall group or time effects were observed, unpaired $t$-test or LSD post hoc test were used to elucidate differences between or within the groups, respectively. Statistical analyses of differences between the two groups for all other parameters pertaining to in vivo pharmacokinetic studies were carried out using unpaired $t$-test. Results from the TST and locomotor studies were analyzed statistically by one-way ANOVA with LSD post hoc. The strength of the association between immobility in the TST and brain escitalopram concentrations was evaluated using the Spearman's rank-order correlation analysis. The criterion for statistical significance was $p \leqslant 0.05$.

\section{RESULTS}

\section{In Vitro Bidirectional Transport Studies}

Western blot analysis. Western blot analysis confirmed that the expression of P-gp in ABCB1-transfected MDCKIIMDR1 cells was higher than endogenous P-gp expression in MDCKII-WT cells (Figure 1b).

Human P-gp limited escitalopram transport across MDCKII-MDR 1 cells. The TR compares drug permeability across the cell monolayer in the basolateral-to-apical $(\mathrm{B} \rightarrow \mathrm{A})$ direction to drug permeability in the apicalto-basolateral direction $(\mathrm{A} \rightarrow \mathrm{B})$. Drugs that are transported substrates of human P-gp will have greater permeability in the $\mathrm{B} \rightarrow \mathrm{A}$ than in the $\mathrm{A} \rightarrow \mathrm{B}$ direction (ie, $\mathrm{TR}>1$ ) in MDCKII-MDR1 cells, due to P-gp efflux restricting drug transport in the $A \rightarrow B$ direction only (Figure 1a). To account for the potential impact of endogenous transporters of MDCK cells, a cTR is determined relative to WT cells.

In MDCKII-WT cells, which are not transfected with $A B C B 1$ and therefore do not express human P-gp, the apparent permeability $\left(\mathrm{P}_{\mathrm{app}}\right)$ of escitalopram was greater in the $\mathrm{B} \rightarrow \mathrm{A}$ than in the $\mathrm{A} \rightarrow \mathrm{B}$ direction, with a TR of 2.32 (Figure 1c and Table 1). This indicates that endogenous MDCK transporters exert a net efflux effect on escitalopram.

In MDCKII-MDR1 cells, which express human P-gp on the apical membrane only (Figure 1a), the difference between $\mathrm{B} \rightarrow \mathrm{A}$ and $\mathrm{A} \rightarrow \mathrm{B} \mathrm{P}_{\text {app }}$ was greater than in MDCKII-WT cells, as evidenced by the TR of 7.22 and cTR of 3.11 (Figure 1d and Table 1). Therefore, both the TR and cTR for escitalopram substantially exceeded the recommended TR and CTR thresholds of 2 and 1.5, respectively, for the identification of transported substrates of human P-gp (Polli et al, 2001; Schwab et al, 2003; Zhang et al, 2006). Moreover, coincubation of the P-gp inhibitor verapamil $(200 \mu \mathrm{M})$ reduced the TR in MDCKII-MDR1 cells to 1.51 (Figure 1e and Table 1). Similarly, coincubation of another P-gp inhibitor, CsA $(25 \mu \mathrm{M})$, reduced the TR in MDCKIIMDR1 cells to 3.33 (Figure if and Table 1). Taken together, the data from this series of in vitro bidirectional transport

Table I Apparent Permeability $\left(\mathrm{P}_{\text {app }}\right)$, Transport Ratio (TR), and Corrected Transport Ratio (cTR) Values from In Vitro Bidirectional Transport Studies

\begin{tabular}{|c|c|c|c|c|c|}
\hline Cell type & Treatment (P-gp inhibitor) & $P_{\text {app }} A \rightarrow B\left(\times 10^{-6} \mathrm{~cm} / \mathrm{s}\right)$ & $P_{\text {app }} B \rightarrow A\left(\times 10^{-6} \mathrm{~cm} / \mathrm{s}\right)$ & TR & cTR \\
\hline MDCKII-WT & None & $7.75 \pm 0.33$ & $|8.00 \pm 0.5|$ & 2.32 & $N / A$ \\
\hline MDCKII-MDRI & None & $2.11 \pm 0.10$ & $|5.23 \pm 0.5|$ & $7.22^{\mathrm{a}}$ & $3.11^{\mathrm{a}}$ \\
\hline MDCKII-MDRI & Verapamil $(200 \mu \mathrm{M})$ & $4.87 \pm 0.65$ & $7.36 \pm 1.13$ & 1.51 & $N / A$ \\
\hline MDCKII-MDRI & CsA $(25 \mu \mathrm{M})$ & $3.35 \pm 0.39$ & $11.12 \pm 0.42$ & 3.33 & $N / A$ \\
\hline
\end{tabular}

aExceeds predefined threshold for identification of a transported P-gp substrate (Polli et al, 200 I; Schwab et al, 2003; Zhang et al, 2006). 
experiments demonstrate that escitalopram is a transported substrate of human P-gp.

\section{In Vivo Pharmacokinetic Studies}

P-gp inhibition had no effect on plasma escitalopram pharmacokinetics. Both CsA- and vehicle-treated groups exhibited similar plasma escitalopram pharmacokinetics, with no group effect on plasma escitalopram levels over time $(\mathrm{F}(1,8)=0.104, p=0.756$; Figure 2a). Steady-state plasma escitalopram levels were reached within $120 \mathrm{~min}$. However, after administration of either vehicle or CsA at $t=120 \mathrm{~min}$, an equivalent $15-20 \%$ increase in plasma escitalopram concentrations, compared to steady-state levels, was evident in both groups. As there was no difference between the groups in terms of plasma escitalopram pharmacokinetics, any differences in brain escitalopram concentrations observed between the groups can be attributed to altered BBB transport.
P-gp inhibition increased escitalopram concentration in microdialysis samples. Steady-state dialysate levels of escitalopram were achieved within $120 \mathrm{~min}$ in all cases. There was a statistically significant difference between the groups in terms of dialysate escitalopram profiles $(\mathrm{F}(1,8)=$ 11.569, $p=0.009$; Figure 2b). There was also a significant overall time effect $(F(7,56)=24.763, p<0.001)$, in addition to a significant group $\times$ time interaction $(\mathrm{F}(1,56)=10.160$, $p<0.001)$.

In the Escit $+\mathrm{VEH}$ group, administration of vehicle resulted in an increase in dialysate escitalopram levels relative to steady state. This increase mirrored the 15-20\% increase evident in plasma levels and reached a maximum $17.08 \%$ at $\mathrm{t}=200 \mathrm{~min}$, thereby indicating that administration of vehicle had no effect on the transport of escitalopram across the $\mathrm{BBB}$. The increase from steady state was statistically significant from $180 \mathrm{~min}$ onwards (Figure $2 \mathrm{~b}$ ).

In the Escit + CsA group, treatment with the P-gp inhibitor CsA resulted in an increase from steady-state
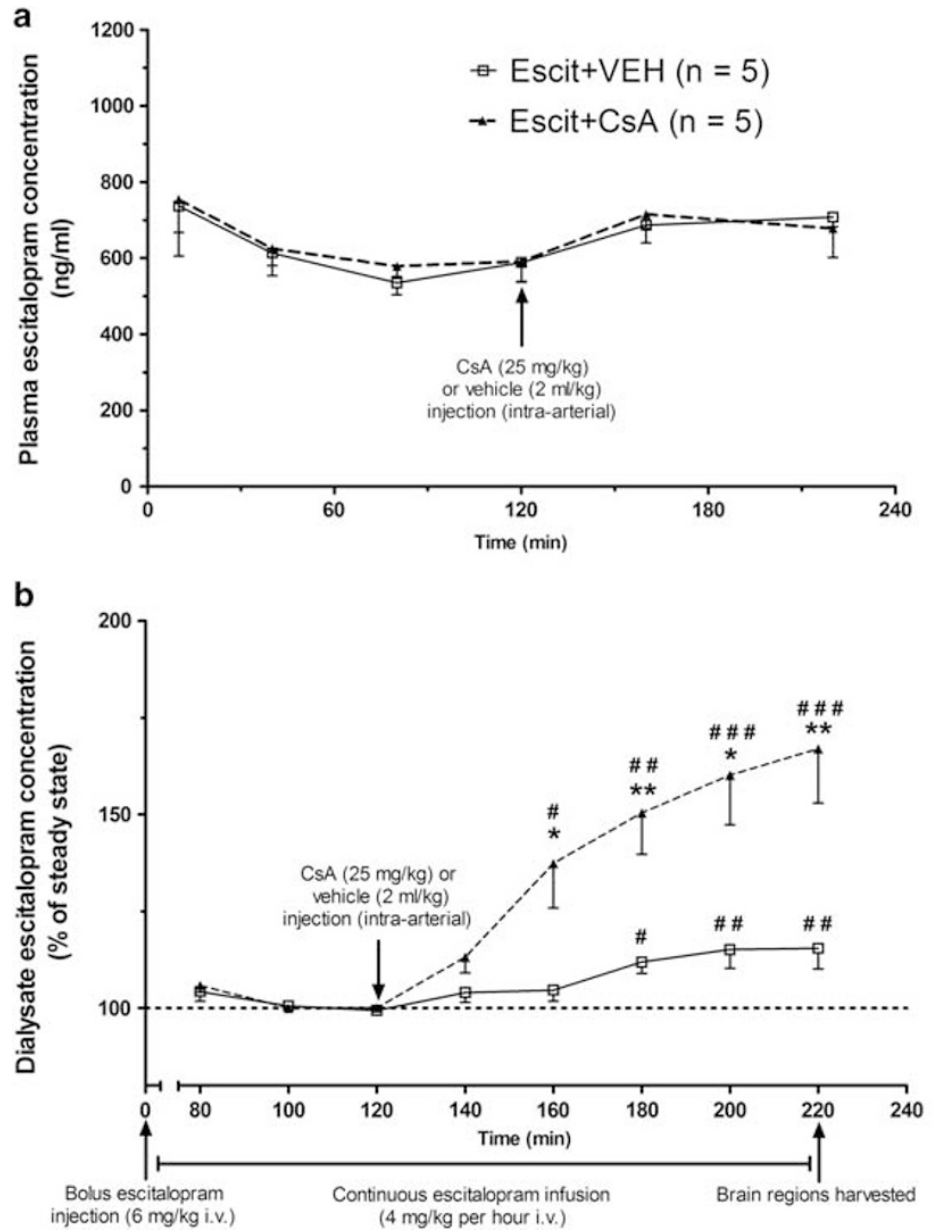

C

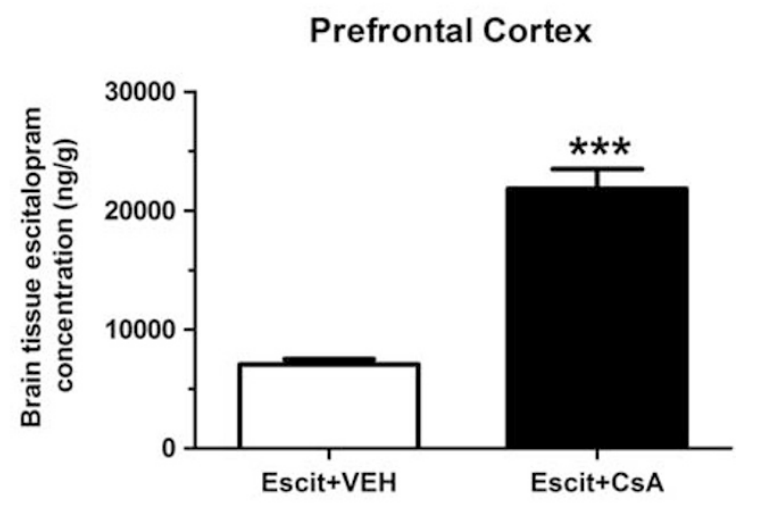

d

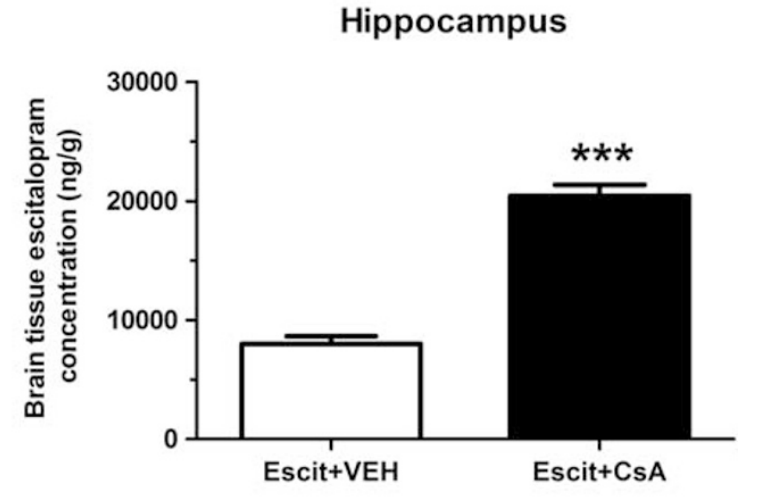

Figure 2 In vivo pharmacokinetic studies. (a) Plasma escitalopram pharmacokinetics. There was no difference in escitalopram plasma pharmacokinetics between vehicle- and cyclosporin A (CsA)-treated animals. Steady-state plasma escitalopram levels were achieved within 120 min in both groups. Administration of vehicle or the P-glycoprotein (P-gP) inhibitor CSA resulted in an equivalent 15-20\% increase in plasma escitalopram levels from steady state in both groups. (b) Dialysate escitalopram pharmacokinetics. Steady-state dialysate concentrations of escitalopram were also achieved in both groups within 120 min. Administration of vehicle resulted in a significant 17\% increase in dialysate escitalopram concentration from steady-state levels, which mirrored increases observed in the plasma. Administration of the P-gp inhibitor CsA resulted in a 67\% increase from steady state, which was significantly greater than that observed in the vehicle-treated group. ( $c$ and d) Brain tissue escitalopram concentrations. Escitalopram concentrations in brain tissue at termination of in vivo pharmacokinetic studies were significantly greater in CsA-treated animals than vehicle-treated animals in both the prefrontal cortex (c) and the hippocampus (d) (mean \pm SEM; $n=5$ per group for all graphs). ${ }^{*} p<0.05 ;{ }^{* *} p<0.01$; ${ }^{* * * *} p<0.00$ I between the groups; ${ }^{\#} p<0.05$; ${ }^{\# \#} p<0.0$ I; $\# \#$ \# $<0.001$ compared with steady-state levels. 
levels, reaching a maximum increase of $66.94 \%$ at $t=220$ min, with a statistically significant elevation relative to steady state from $t=160 \mathrm{~min}$ onwards (Figure $2 \mathrm{~b}$ ). Moreover, the increase in the Escit + CsA group was significantly greater than that observed in the Escit + VEH group from $t=160 \mathrm{~min}$ onwards (Figure 2b). Taken together, these in vivo plasma and microdialysis pharmacokinetic data demonstrate that inhibition of P-gp results in enhanced BBB transport of escitalopram.

Brain tissue escitalopram concentrations were increased following P-gp inhibition. At termination of the microdialysis experiment, PFC brain tissue escitalopram concentrations were over three times greater in CsA-treated animals than vehicle-treated controls $(t(8)=8.602$, $p<0.001$; Figure 2c). Moreover, the PFC brain tissue: plasma escitalopram concentration ratio was also elevated over threefold in CsA-treated animals relative to controls $(t(5.617)=5.617, p=0.004$; Table 2$)$, thus demonstrating that escitalopram transport across the BBB is significantly increased by P-gp inhibition.

Similarly, hippocampal brain tissue escitalopram concentrations were more than 2.5 times greater in CsA-treated animals than vehicle-treated controls $(t(8)=11.047$, $p<0.001$; Figure 2d). In addition, the hippocampal brain tissue: plasma escitalopram concentration ratio was elevated more than 2.75 -fold in CsA-treated animals relative to vehicle-treated controls $(t(8)=5.458, p=0.001$; Table 2$)$. These findings corroborate the microdialysis results, and demonstrate that inhibition of P-gp results in enhanced transport of escitalopram across the BBB.

\section{In Vivo Pharmacodynamic Studies}

Verapamil augmented escitalopram response in TST. The TST is one of the most widely used models for the assessment of antidepressant activity in mice (Cryan et al, 2005). When subjected to the short-term inescapable stress of suspension by the tail, mice adopt an immobile posture. Treatment with antidepressant medications, including escitalopram (Zomkowski et al, 2010), reduces the time spent immobile in this paradigm in a dose-responsive manner (Cryan et al, 2005). In this study, it was found that pre-treatment with the P-gp inhibitor verapamil augmented the antidepressant response at both doses of escitalopram investigated (Figure 3a). There was a significant overall difference between the groups in immobility in the TST $(\mathrm{F}(5,61)=6.964, p<0.001$; Figure 3a). Post hoc analysis revealed that the 23-s reduction in immobility, compared to control animals, observed in mice treated with the lower dose of escitalopram $(0.1 \mathrm{mg} / \mathrm{kg})$ without verapamil pre-treatment was not statistically significant $(p=0.168)$. Pre-treatment with verapamil augmented the response to this lower dose of escitalopram by over $60 \%$, resulting in a statistically significant 38 - $s$ reduction in immobility vs controls $(p=0.01)$. Moreover, while treatment with the higher dose of escitalopram $(1 \mathrm{mg} / \mathrm{kg})$ led to a significant reduction in immobility with or without pretreatment with verapamil $(p=0.001$ and $<0.001$ respectively), the magnitude of the reduction was augmented by almost $40 \%$ following pre-treatment with verapamil (Figure 3a). These results demonstrate that inhibition of P-gp augments the antidepressant-like effect of escitalopram in the TST.

Effects of escitalopram and verapamil in the TST were not a function of increased locomotor activity. Treatments that lead to increased locomotor activity could cause a reduction in immobility in the TST unrelated to antidepressant-like activity. To investigate this potential confounding factor, the effects of the present drug treatments on locomotor activity were assessed. There was a significant difference in locomotor activity between the groups $(\mathrm{F}(3,23)=4.605, p=0.012$; Figure $3 \mathrm{~b})$. Post hoc analysis revealed that mice in receipt of pre-treatment with verapamil exhibited decreased locomotion relative to saline-treated controls, whether subsequently treated with saline $(p=0.016)$ or escitalopram $(p=0.002)$. Administration of escitalopram without verapamil pre-treatment had no effect on locomotor activity $(p=0.252)$. As locomotor activity was not increased by treatment with verapamil and/or escitalopram, the decreases in immobility observed in the TST can be attributed to the antidepressant-like activity of escitalopram, as opposed to being an artifact of increased locomotion.

Brain tissue concentrations of escitalopram were increased following verapamil pre-treatment. Brain tissue escitalopram concentrations were significantly greater in mice pre-treated with the P-gp inhibitor verapamil than

Table 2 Escitalopram Concentrations in Plasma and Brain Tissue, and Brain Tissue: Plasma Escitalopram Concentration Ratios at Termination of In Vivo Pharmacokinetic Studies

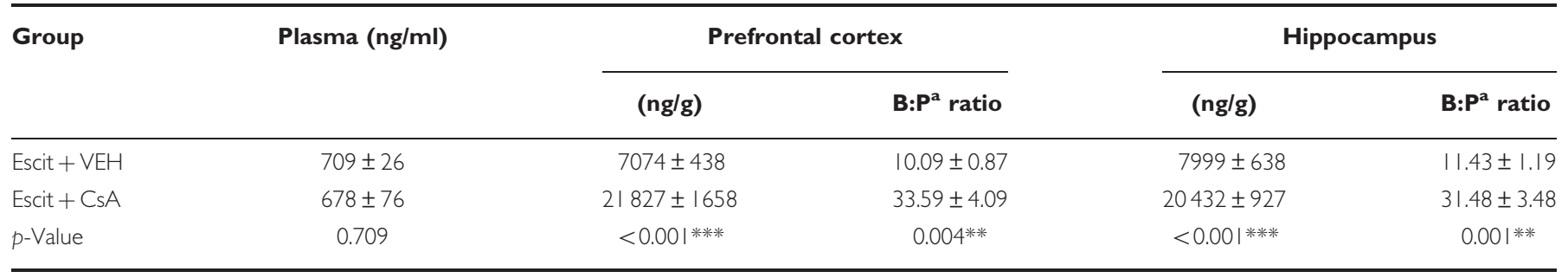

$n=5$ per group.

*** $p<0.01$.

**** $p<0.001$.

${ }^{a}$ Brain region: plasma ratio. 
a

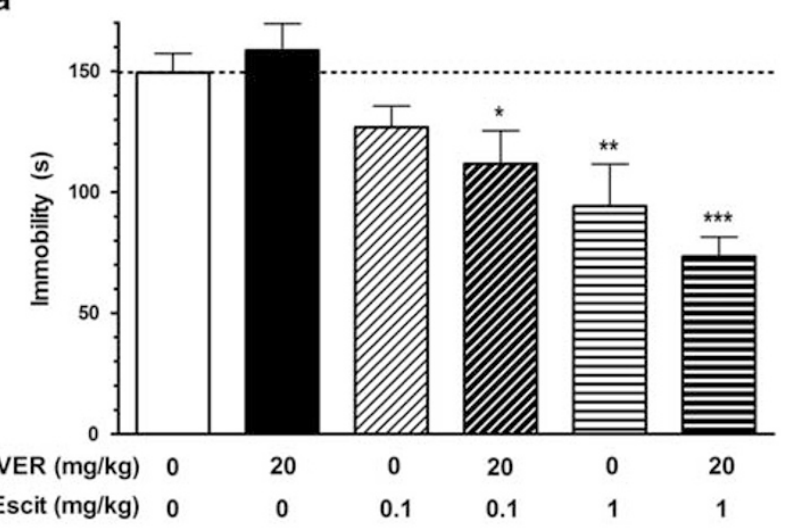

b

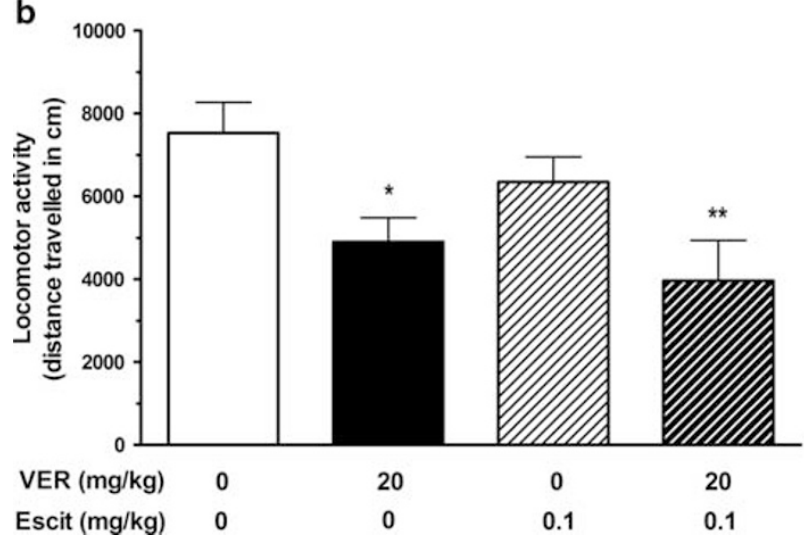

C

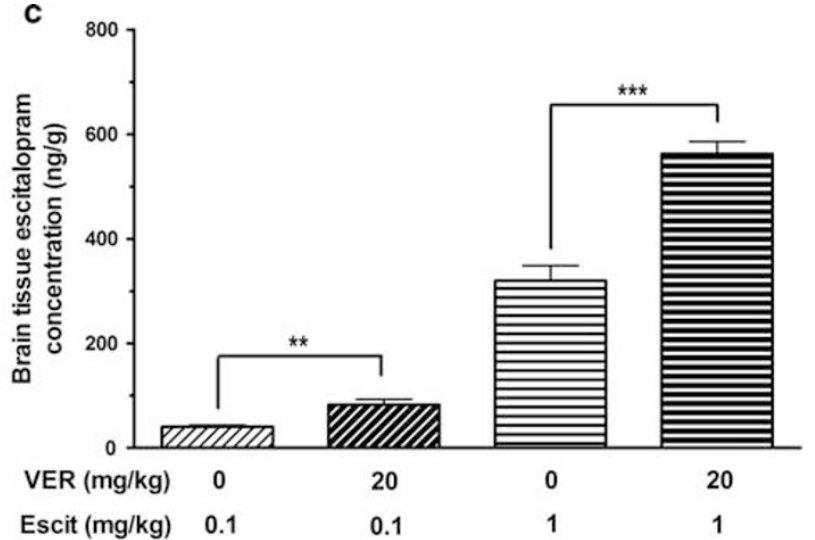

d

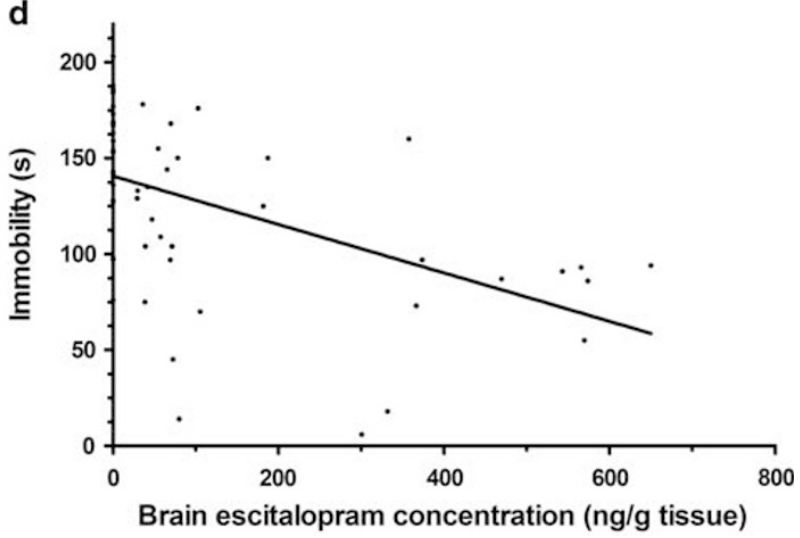

Figure 3 In vivo pharmacodynamic studies. (a) Tail suspension test. Treatment with escitalopram reduced the time spent immobile in the tail suspension test (TST) in a dose-dependent manner. Treatment with verapamil without escitalopram had no impact on immobility. However, pre-treatment with verapamil augmented the antidepressant-like effect of escitalopram in the TST, further reducing the time spent immobile at both doses of escitalopram (mean \pm SEM; $n=8-17$ per group). (b) Locomotor activity. Treatment with verapamil, escitalopram, or both did not increase locomotor activity, indicating that the reductions in immobility observed in the TST were due to antidepressant-like effects. In fact, treatment with verapamil reduced locomotor activity, with or without escitalopram treatment (mean \pm SEM; $n=6-7$ per group). (c) Brain tissue escitalopram concentrations. Pre-treatment with the P-gP inhibitor verapamil before administration of escitalopram significantly increased brain concentrations of escitalopram in mice used in the TST at both doses of escitalopram treatment (mean \pm SEM; $n=6-12$ per group). (d) Correlation between brain escitalopram concentration and immobility in the TST. There was a moderately strong negative correlation between escitalopram concentration in brain tissue and immobility in the TST $\left(r_{\mathrm{s}}=-0.594, n=56, p<0.00 \mathrm{I}\right)$. ${ }^{*} p<0.05$; ** $p<0.01$; **** $<0.00$ I relative to saline-saline control group (or between two groups, where indicated in (c)).

control mice at both doses of escitalopram treatment (Figure 3c). Pre-treatment with verapamil resulted in a $>2$-fold increase in brain escitalopram levels in mice treated with $0.1 \mathrm{mg} / \mathrm{kg}$ of escitalopram $(t(17)=-3.010$, $p=0.008)$ and a $>1.75$-fold increase in brain escitalopram levels in mice treated with $1 \mathrm{mg} / \mathrm{kg}$ of escitalopram $(t(10)=-6.516, p<0.001)$. Verapamil concentration in brain tissue was consistent across the three verapamiltreated groups $(1163 \pm 53 \mathrm{ng} / \mathrm{ml})$. These results confirm that administration of verapamil as a P-gp inhibitor resulted in increased brain levels of escitalopram in the mice during the TST.

Correlation between brain escitalopram concentration and immobility in the TST. There was a moderately strong and statistically significant negative correlation between escitalopram concentration in brain tissue and immobility in the TST $\left(r_{s}=-0.594, n=56, p<0.001\right.$; Figure 3d). This indicates that the decreased immobility observed in escitalopram-treated mice in receipt of verapamil pre-treatment was related to the increased delivery of escitalopram to the brains of these animals.

\section{DISCUSSION}

These findings clearly demonstrate that P-gp modulates the pharmacokinetics and pharmacodynamics of the widely prescribed antidepressant escitalopram. In showing that escitalopram is a transported substrate of human P-gp, and that pharmacological inhibition of $\mathrm{P}$-gp results in enhanced escitalopram transport across the $\mathrm{BBB}$ in vivo, we open up the concept that P-gp inhibition may be a viable and potentially safe strategy to augment the effects of escitalopram. Moreover, we bolster this hypothesis by showing a pharmacodynamic consequence of elevated brain levels of escitalopram by P-gp inhibition in a relevant animal model of antidepressant activity. These novel findings are especially important in light of recent clinical studies, which have found associations between SNPs in $A B C B 1$ and response to escitalopram treatment (Lin et al, 2011) and the 
dose of escitalopram required to achieve remission in major depression (Singh et al, 2012). Although there is generally no clear correlation between antidepressant plasma levels and therapeutic efficacy (Perry et al, 1987; Spina et al, 1997; Normann et al, 2004), it has been proposed that interindividual variability in $\mathrm{BBB}$ transport, due to genetic differences in P-gp functionality, may lead to clinically relevant differences in the brain distribution of certain antidepressants (Kato et al, 2008). These results, in conjunction with previously reported pharmacogenetic associations between $A B C B 1$ genotype and treatment response (Lin et al, 2011; Singh et al, 2012), support this hypothesis in relation to escitalopram, indicating that $\mathrm{P}$-gp may prevent therapeutic brain concentrations from being achieved in certain patients with elevated P-gp activity due to functional SNPs in $A B C B 1$.

To our knowledge, the behavioral studies included in this work demonstrate for the first time that the pharmacodynamic activity of an antidepressant can be augmented by inhibition of P-gp. In addition, analysis of the correlation between time spent immobile in the TST and brain escitalopram concentrations indicated that the reduction in immobility observed following P-gp inhibition was related to the enhanced delivery of escitalopram to the brain. These novel findings are especially exciting as they indicate that this approach to increase escitalopram concentrations in the brain may prove to be therapeutically beneficial in patients. In previous preclinical studies, escitalopram has been shown to reduce immobility in the TST in a dose-responsive manner up to a maximal response at $1 \mathrm{mg} / \mathrm{kg}$ (Zomkowski et al, 2010). At higher doses of escitalopram, the time spent immobile increased, likely due to peripheral side effects, resulting in a U-shaped doseresponse curve (Zomkowski et al, 2010). In this study, it was found that pre-treatment with the P-gp inhibitor verapamil augmented the antidepressant response at both doses of escitalopram investigated, including an enhanced response to a $1 \mathrm{mg} / \mathrm{kg}$ dose (Figure 3a). This highlights that the P-gp inhibition strategy may facilitate augmentation of the central effects of escitalopram while avoiding peripheral side effects associated with higher doses of escitalopram. However, it should be noted that this approach could potentially result in increased risk of unwanted central side effects. This risk could be offset by selectively using the P-gp inhibition augmentation strategy in patients shown to have enhanced P-gp functionality or in conjunction with low-dose escitalopram, thereby serving to normalize brain levels of escitalopram rather than resulting in toxic brain concentrations.

Our finding that administration of a P-gp inhibitor increased brain concentrations of escitalopram without altering plasma levels demonstrates that inhibition of P-gp enhances escitalopram transport across the $\mathrm{BBB}$. Importantly, the intracerebral microdialysis technique employed to monitor brain levels of escitalopram measures only unbound drug levels in brain extracellular fluid (de Lange et al, 2000). Given that drugs are active in the unbound form and that escitalopram acts within the brain by inhibiting the serotonin reuptake transporter in the extracellular synaptic cleft, these findings are of particular pharmacological relevance. Furthermore, the steady-state approach adopted in this study enabled the confirmation of microdialysis findings by determining brain tissue concentrations from the same animals at termination of the experiment, when differences between the groups were maximal. To our knowledge, there are no data available in relation to brain levels of escitalopram in humans in clinical practice. Therefore, it is not possible to discuss the brain concentrations observed in these studies in the context of those encountered clinically. Future imaging or postmortem studies may offer further information in this regard. Nonetheless, our finding that brain tissue levels of escitalopram were increased three fold by P-gp inhibition compares favorably with recently reported results from studies using P-gp knockout mice, which were published online as this paper was being finalized (Karlsson et al, 2013). Given that it has proven difficult to reproduce effects observed in P-gp knockout mice by P-gp inhibition in the past (Ejsing and Linnet, 2005), it remained vital to demonstrate that P-gp inhibition would result in similarly increased brain levels of escitalopram before proposing that coadministration of a P-gp inhibitor may represent a promising approach to enhance escitalopram delivery to the brain. Considering that patients are treated chronically with antidepressants in clinical practice, future studies are required to investigate if escitalopram concentrations in the brain can be enhanced and maintained by repeated P-gp inhibition.

Well-documented interspecies differences in P-gp substrate specificity (Yamazaki et al, 2001; Katoh et al, 2006; Baltes et al, 2007; Syvanen et al, 2009) mean that findings in relation to P-gp transport obtained from animal models cannot be readily extrapolated to humans. Therefore, appropriate in vitro bidirectional transport studies involving human P-gp are necessary to enable evaluation of the clinical relevance of results from rodent studies. Indeed, this is a very pertinent point in light of our recent findings that the antidepressants amitriptyline and fluoxetine, both of which have been identified as P-gp substrates in mice (Uhr et al, 2000; Doran et al, 2005), are not transported substrates of human P-gp (O'Brien et al, 2013). Therefore, the present study's finding that escitalopram is a transported substrate of human P-gp indicates that results from our in vivo studies may translate to humans.

Although these studies have exclusively involved escitalopram, it is possible that our findings may extend to certain other antidepressant drugs, as there is a growing body of evidence to suggest that efflux by P-gp may influence treatment response to several antidepressants (reviewed in O'Brien et al, 2012b). There is limited information available in relation to antidepressant transport by human P-gp per se. However, in common with escitalopram, pharmacogenetic studies have revealed an association between SNPs in $A B C B 1$ and response to other antidepressants (Gex-Fabry et al, 2008; Kato et al, 2008; Nikisch et al, 2008; Uhr et al, 2008; Sarginson et al, 2010). These antidepressants, including citalopram and paroxetine, are thought to be P-gp substrates based on results from studies in P-gp knockout mice (Uhr and Grauer, 2003; Uhr et al, 2003, 2008; Doran et al, 2005). However, it has been reported that similar pharmacogenetic associations do not exist for other antidepressants, including amitriptyline, duloxetine, fluoxetine, and mirtazapine (Roberts et al, 2002; Laika et al, 2006; Uhr et al, 2008; Perlis et al, 2010). 
Emerging data from our lab, indicating that these four antidepressants are not transported substrates of human P-gp, despite aforementioned preclinical findings in relation to amitriptyline and fluoxetine (Uhr et al, 2000; Doran et al, 2005), may offer an explanation for these observations (O'Brien et al, 2013). Further studies are now warranted to determine if the present study's findings in relation to escitalopram generalize to other antidepressants.

It should be noted that transporters other than P-gp, such as breast cancer resistance protein (BCRP) and the multidrug resistance-associated proteins (MRPs), also have an important role at the BBB. Given the significant overlap in substrate specificity between P-gp, BCRP and the MRPs (Sharom, 2008), it is possible that these alternative transporters may also influence the distribution of escitalopram across the $\mathrm{BBB}$. Indeed, racemic citalopram has been reported to be an MRP1 substrate, while the same study found an association between polymorphisms in the MRP1 gene and citalopram response (Lee et al, 2010). Nonetheless, the range of approaches adopted in these studies, not least the in vitro study involving MDCKIIMDR1 cells transfected with human P-gp, highlight an important role for P-gp in escitalopram pharmacokinetics and pharmacodynamics independent of any potential involvement of other transporters.

In summary, these studies indicate that $\mathrm{P}$-gp may restrict the $\mathrm{BBB}$ transport of escitalopram in humans, potentially resulting in subtherapeutic brain concentrations in certain patients, particularly those with elevated P-gp functionality because of SNPs in $A B C B 1$. Moreover, the novel finding that increasing escitalopram delivery to the brain by P-gp inhibition results in enhanced antidepressant-like activity indicates that adjunctive treatment with a P-gp inhibitor may represent a beneficial approach to augment escitalopram therapy in depression in such patients. This approach could potentially have the added benefit of reducing the administered dose required to achieve escitalopram response, which is particularly desirable following recent guidance recommending the limitation of escitalopram doses used clinically because of the risk of QT prolongation (Medicines and Healthcare products Regulatory Agency, 2011). The P-gp inhibitors verapamil and CsA, used as tool compounds in the present proof-of-concept study, have the advantage of being clinically available for non-CNS indications. However, it is unlikely that these drugs would be appropriate for clinical use as P-gp inhibitors because of their lack of specificity and potency, thereby requiring high doses to adequately inhibit P-gp. Verapamil is primarily used as a calcium channel blocker, whereas CsA is an immunosuppressant agent, which also modulates other drug transporters (Qadir et al, 2005). The use of more selective second- or third-generation P-gp inhibitors may represent a more acceptable alternative for potential clinical use. Additional studies, both preclinical and clinical, are now warranted to further evaluate the safety and efficacy of this strategy to augment treatment response to escitalopram.

\section{FUNDING AND DISCLOSURE}

The authors declare no conflict of interest.

\section{ACKNOWLEDGEMENTS}

We thank Patrick Fitzgerald, Rachel Moloney, Lieve Desbonnet, Caroll Beltrán, Maria Donovan, and staff in the University College Cork Biological Services Unit for technical assistance with this study. The Alimentary Pharmabiotic Center is a research center funded by Science Foundation Ireland (SFI), through the Irish Government's National Development Plan. TGD and JFC are supported by SFI (Grant Nos. 02/CE/B124 and 07/CE/B1368). The SFI-funded Strategic Research Cluster Grant No. 07/SRC/B1154 and the Irish Drug Delivery Network also fund BTG and JFC. TD, JFC, and GC are also supported by the Irish Health Research Board Health Research Awards (HRA_POR/2011/23) and (HRA_POR/2012/32). GC is in receipt of research funding from the American Neurogastroenterology and Motility Society (ANMS).

\section{REFERENCES}

Baltes S, Gastens AM, Fedrowitz M, Potschka H, Kaever V, Loscher W (2007). Differences in the transport of the antiepileptic drugs phenytoin, levetiracetam and carbamazepine by human and mouse P-glycoprotein. Neuropharmacology 52: 333-346.

Bundgaard C, Jensen CJ, Garmer M (2012). Species comparison of in vivo P-glycoprotein-mediated brain efflux using mdrladeficient rats and mice. Drug Metab Dispos 40: 461-466.

Bundgaard C, Jorgensen M, Mork A (2007). An integrated microdialysis rat model for multiple pharmacokinetic/pharmacodynamic investigations of serotonergic agents. J Pharmacol Toxicol Methods 55: 214-223.

Clarke G, O'Mahony SM, Cryan JF, Dinan TG (2009). Verapamil in treatment resistant depression: a role for the P-glycoprotein transporter? Hum Psychopharmacol 24: 217-223.

Cryan JF, Mombereau C, Vassout A (2005). The tail suspension test as a model for assessing antidepressant activity: review of pharmacological and genetic studies in mice. Neurosci Biobehav Rev 29: 571-625.

de Lange EC, de Boer AG, Breimer DD (2000). Methodological issues in microdialysis sampling for pharmacokinetic studies. Adv Drug Deliv Rev 45: 125-148.

Doran A, Obach RS, Smith BJ, Hosea NA, Becker S, Callegari E et al (2005). The impact of P-glycoprotein on the disposition of drugs targeted for indications of the central nervous system: evaluation using the MDR1A/1B knockout mouse model. Drug Metab Dispos 33: 165-174.

Ejsing TB, Linnet K (2005). Influence of P-glycoprotein inhibition on the distribution of the tricyclic antidepressant nortriptyline over the blood-brain barrier. Hum Psychopharmacol 20: 149-153.

Frahnert C, Rao ML, Grasmader K (2003). Analysis of eighteen antidepressants, four atypical antipsychotics and active metabolites in serum by liquid chromatography: a simple tool for therapeutic drug monitoring. J Chromatogr B 794: 35-47.

Gex-Fabry M, Eap CB, Oneda B, Gervasoni N, Aubry JM, Bondolfi G et al (2008). CYP2D6 and ABCB1 genetic variability: influence on paroxetine plasma level and therapeutic response. Ther Drug Monit 30: 474-482.

Karlsson L, Carlsson B, Hiemke C, Ahlner J, Bengtsson F, Schmitt $\mathrm{U}$ et al (2013). Altered brain concentrations of citalopram and escitalopram in P-glycoprotein deficient mice after acute and chronic treatment. Eur Neuropsychopharmacol (doi:10.1016/j. euroneuro.2013.01.003.

Karlsson L, Schmitt U, Josefsson M, Carlsson B, Ahlner J, Bengtsson F et al (2010). Blood-brain barrier penetration of the enantiomers of venlafaxine and its metabolites in mice lacking P-glycoprotein. Eur Neuropsychopharmacol 20: 632-640. 
Kato M, Fukuda T, Serretti A, Wakeno M, Okugawa G, Ikenaga Y et al (2008). ABCB1 (MDR1) gene polymorphisms are associated with the clinical response to paroxetine in patients with major depressive disorder. Prog Neuro-Psychopharamacol 32: 398-404.

Katoh M, Suzuyama N, Takeuchi T, Yoshitomi S, Asahi S, Yokoi T (2006). Kinetic analyses for species differences in P-glycoprotein-mediated drug transport. J Pharm Sci 95: 2673-2683.

Kennedy SH, Andersen HF, Lam RW (2006). Efficacy of escitalopram in the treatment of major depressive disorder compared with conventional selective serotonin reuptake inhibitors and venlafaxine XR: a meta-analysis. J Psychiatry Neurosci 31: 122-131.

Kuteykin-Teplyakov K, Luna-Tortos C, Ambroziak K, Loscher W (2010). Differences in the expression of endogenous efflux transporters in MDR1-transfected versus wildtype cell lines affect P-glycoprotein mediated drug transport. $\mathrm{Br} J$ Pharmacol 160: 1453-1463.

Laika B, Leucht S, Steimer W (2006). ABCB1 (P-Glycoprotein/ MDR1) Gene G2677T/A sequence variation (polymorphism): lack of association with side effects and therapeutic response in depressed inpatients treated with amitriptyline. Clin Chem 52: 893-895.

Lee SH, Lee MS, Lee JH, Kim SW, Kang RH, Choi MJ et al (2010). MRP1 polymorphisms associated with citalopram response in patients with major depression. J Clin Psychopharm 30: 116-125.

Lin KM, Chiu YF, Tsai IJ, Chen CH, Shen WW, Liu SC et al (2011). $\mathrm{ABCB} 1$ gene polymorphisms are associated with the severity of major depressive disorder and its response to escitalopram treatment. Pharmacogenet Genom 21: 163-170.

Loscher W, Potschka H (2005). Drug resistance in brain diseases and the role of drug efflux transporters. Nat Rev Neurosci 6: 591-602.

Medicines and Healthcare Products Regulatory Agency (2011): Citalopram and escitalopram: QT interval prolongationnew maximum daily dose restrictions (including in elderly patients), contraindications, and warnings http://www.mhra.gov. uk/Safetyinformation/DrugSafetyUpdate/CON137769.

Nikisch G, Eap CB, Baumann P (2008). Citalopram enantiomers in plasma and cerebrospinal fluid of $\mathrm{ABCB} 1$ genotyped depressive patients and clinical response: a pilot study. Pharmacol Res 58: 344-347.

Normann C, Horn M, Hummel B, Grunze H, Walden R (2004). Paroxetine in major depression: correlating plasma concentrations and clinical response. Pharmacopsychiatry 37: 123-126.

O’Brien FE, Clarke G, Dinan TG, Cryan JF, Griffin BT (2013). Human P-glycoprotein differentially affects antidepressant drug transport: relevance to blood-brain barrier permeability. Int $J$ Neuropsychopharmacol.

O’Brien FE, Clarke G, Fitzgerald P, Dinan TG, Griffin BT, Cryan JF (2012a). Inhibition of P-glycoprotein enhances transport of imipramine across the blood-brain barrier: microdialysis studies in conscious freely moving rats. Br J Pharmacol 166: 1333-1343.

O’Brien FE, Dinan TG, Griffin BT, Cryan JF (2012b). Interactions between antidepressants and P-glycoprotein at the blood-brain barrier: clinical significance of in vitro and in vivo findings. Br J Pharmacol 165: 289-312.

O'Connor RM, Cryan JF (2013). The effects of mGlu7 receptor modulation in behavioural models sensitive to antidepressant action in two mouse strains. Behav Pharmacol 24: 105-113.

Pastan I, Gottesman MM, Ueda K, Lovelace E, Rutherford AV, Willingham MC (1988). A retrovirus carrying an MDR1 cDNA confers multidrug resistance and polarized expression of P-glycoprotein in MDCK cells. Proc Natl Acad Sci USA 85: 4486-4490.

Paxinos G, Watson C (1998). The Rat Brain in Stereotaxic Coordinates. 4th edn. Academic Press: San Diego, CA, USA.

Perlis RH, Fijal B, Dharia S, Heinloth AN, Houston JP (2010). Failure to replicate genetic associations with antidepressant treatment response in duloxetine-treated patients. Biol Psychiatry 67: 1110-1113.
Perry PJ, Pfohl BM, Holstad SG (1987). The relationship between antidepressant response and tricyclic antidepressant plasma concentrations. A retrospective analysis of the literature using logistic regression analysis. Clin Pharmacokinet 13: 381-392.

Polli JW, Wring SA, Humphreys JE, Huang L, Morgan JB, Webster LO et al (2001). Rational use of in vitro P-glycoprotein assays in drug discovery. J Pharmacol Exp Ther 299: 620-628.

Qadir M, O'Loughlin KL, Fricke SM, Williamson NA, Greco WR, Minderman $\mathrm{H}$ et al (2005). Cyclosporin A is a broad-spectrum multidrug resistance modulator. Clin Cancer Res 11: 2320-2326.

Roberts RL, Joyce PR, Mulder RT, Begg EJ, Kennedy MA (2002). A common P-glycoprotein polymorphism is associated with nortriptyline-induced postural hypotension in patients treated for major depression. Pharmacogenom J 2: 191-196.

Sarginson JE, Lazzeroni LC, Ryan HS, Ershoff BD, Schatzberg AF, Murphy GM (2010). ABCB1 (MDR1) polymorphisms and antidepressant response in geriatric depression. Pharmacogenet Genom 20: 467-475.

Schwab D, Fischer H, Tabatabaei A, Poli S, Huwyler J (2003). Comparison of in vitro P-glycoprotein screening assays: recommendations for their use in drug discovery. J Med Chem 46: 1716-1725.

Sharom FJ (2008). ABC multidrug transporters: structure, function and role in chemoresistance. Pharmacogenomics 9: 105-127.

Singh AB, Bousman CA, Ng CH, Byron K, Berk M (2012). ABCB1 polymorphism predicts escitalopram dose needed for remission in major depression. Transl Psychiatry 2: e198.

Spina E, Gitto C, Avenoso A, Campo GM, Caputi AP, Perucca E (1997). Relationship between plasma desipramine levels, CYP2D6 phenotype and clinical response to desipramine: a prospective study. Eur J Clin Pharmacol 51: 395-398.

Syvanen S, Lindhe O, Palner M, Kornum BR, Rahman O, Langstrom B et al (2009). Species differences in blood-brain barrier transport of three positron emission tomography radioligands with emphasis on P-glycoprotein transport. Drug Metab Dispos 37: 635-643.

Tang F, Horie K, Borchardt RT (2002). Are MDCK cells transfected with the human MDR1 gene a good model of the human intestinal mucosa? Pharm Res 19: 765-772.

Taub ME, Podila L, Ely D, Almeida I (2005). Functional assessment of multiple P-glycoprotein (P-gp) probe substrates: influence of cell line and modulator concentration on P-gp activity. Drug Metab Dispos 33: 1679-1687.

Uhr M, Grauer MT (2003). abcblab P-glycoprotein is involved in the uptake of citalopram and trimipramine into the brain of mice. J Psychiat Res 37: 179-185.

Uhr M, Grauer MT, Holsboer F (2003). Differential enhancement of antidepressant penetration into the brain in mice with abcblab (mdrlab) P-glycoprotein gene disruption. Biol Psychiatry 54: $840-846$.

Uhr M, Grauer MT, Yassouridis A, Ebinger M (2007). Blood-brain barrier penetration and pharmacokinetics of amitriptyline and its metabolites in p-glycoprotein (abcblab) knock-out mice and controls. J Psychiatr Res 41: 179-188.

Uhr M, Steckler T, Yassouridis A, Holsboer F (2000). Penetration of amitriptyline, but not of fluoxetine, into brain is enhanced in mice with blood-brain barrier deficiency due to Mdr1a P-glycoprotein gene disruption. Neuropsychopharmacol 22: 380-387.

Uhr M, Tontsch A, Namendorf C, Ripke S, Lucae S, Ising M et al (2008). Polymorphisms in the drug transporter gene ABCB1 predict antidepressant treatment response in depression. Neuron 57: 203-209.

Unceta N, Goicolea MA, Barrio RJ (2011). Analytical procedures for the determination of the selective serotonin reuptake inhibitor antidepressant citalopram and its metabolites. Biomed Chromatogr 25: 238-257.

Yamazaki M, Neway WE, Ohe T, Chen IW, Rowe JF, Hochman JH et al (2001). In vitro substrate identification studies for 


\section{P-glycoprotein and escitalopram}

P-glycoprotein-mediated transport: species difference and predictability of in vivo results. J Pharmacol Exp Ther 296: 723-735. Zhang L, Strong JM, Qiu W, Lesko LJ, Huang SM (2006). Scientific perspectives on drug transporters and their role in drug interactions. Mol Pharmaceut 3: 62-69.
Zomkowski AD, Engel D, Gabilan NH, Rodrigues AL (2010). Involvement of NMDA receptors and L-arginine-nitric oxidecyclic guanosine monophosphate pathway in the antidepressantlike effects of escitalopram in the forced swimming test. Eur Neuropsychopharmacol 20: 793-801.

Supplementary Information accompanies the paper on the Neuropsychopharmacology website (http://www.nature.com/npp) 